\title{
Protective Effect of Molecular Hydrogen Following Different Routes of Administration on D-Galactose- Induced Aging Mice
}

\author{
Boyan Liu $\mathbb{1}^{1-3, *}$ \\ Yunbo $\mathrm{Xie}^{2,4, *}$ \\ Jun Chen ${ }^{4}$ \\ Junli Xue ${ }^{l-3}$ \\ Xiaoyi Zhang ${ }^{2}$ \\ Min Zhao ${ }^{2}$ \\ Xiubin Jia ${ }^{2}$ \\ Yiwei Wang 4 \\ Shucun Qin ${ }^{1,2}$

\begin{abstract}
'The Second Affiliated Hospital of Shandong First Medical University, Tai'an, 27I000, People's Republic of China;

${ }^{2}$ Taishan Institute for Hydrogen Biomedicine, Shandong First Medical University and Shandong Academy of Medical Sciences, Tai'an, 27I000, People's Republic of China; ${ }^{3}$ College of Basic Medical Sciences, Shandong First Medical University and Shandong Academy of Medical Sciences, Jinan, 250II7, People's Republic of China; ${ }^{4}$ Department of General Practice, The Affiliated Hospital of Chengde Medical University, Chengde, 067000, People's Republic of China
\end{abstract} \\ *These authors contributed equally to
} this work

Correspondence: Shucun Qin The Second Affiliated Hospital of Shandong First Medical University, Tai'an, 271000, People's Republic of China $\mathrm{Tel} / \mathrm{Fax}+865386222986$

Email scqin@sdfmu.edu.cn

Yiwei Wang

Department of General Practice, The

Affiliated Hospital of Chengde Medical

University, Chengde, 067000, People's

Republic of China

Tel/Fax +86 31422795 II

Email chengdewyw@126.com
Background: Molecular hydrogen $\left(\mathrm{H}_{2}\right)$ has been recognized as an effective antioxidant with no or little side effects. While it is known that oxidative stress is closely associated with aging, the beneficial effect of $\mathrm{H}_{2}$ on oxidative stress-related aging is still unclear. In this study, a mouse model of D-galactose-induced aging was employed to investigate the protective effects of $\mathrm{H}_{2}$.

Methods: The mice were administrated of $\mathrm{H}_{2}$ via different routes $\left(4 \% \mathrm{H}_{2}\right.$ inhalation, $\mathrm{H}_{2-}$ rich water drinking, and $\mathrm{H}_{2}$-rich saline injection), the aging-related biomarkers in plasma and the oxidative stress in different tissues were measured.

Results: The results showed that $\mathrm{H}_{2}$ improved aging-related biomarkers, ie, total antioxidant capacity, advanced glycation end products, tumor necrosis factor- $\alpha$, free fatty acids, and alanine aminotransferase in plasma. Furthermore, $\mathrm{H}_{2}$ alleviated oxidative stress in the liver, brain, and heart by reducing the levels of lipid peroxidation and malondialdehyde and increasing the activity of superoxide dismutase. In addition, it seems that $4 \% \mathrm{H}_{2}$ inhalation was the most effective regarding the amount of $\mathrm{H}_{2}$ taken up and in reducing the markers of oxidative stress in some of the tissues; however, the other routes of administration resulted in the same efficacy in most indicators.

Conclusion: $\mathrm{H}_{2}$ can prevent oxidative stress in D-galactose-induced aging mice when administered by different routes.

Keywords: molecular hydrogen, administration routes, oxidative stress, aging, D-galactose

\section{Introduction}

Aging is a multifactorial process characterized by the progressive loss of physiological functions that are closely associated with a diversity of chronic diseases. The mechanism of aging is still not clear, although many theories have been proposed to explain this process. ${ }^{1}$ Among them, the free radical/oxidative stress theory of aging has been receiving considerable attention. This theory postulates that aging is caused by cumulative oxidative damage to cells and tissues by reactive oxygen species (ROS). ${ }^{2}$ Oxidative stress occurs when the natural production of ROS cannot be balanced by the antioxidative capacity of tissues, with consequent excessive free radicals causing lipid peroxidation, protein oxidation, DNA damage, and mitochondrial dysfunction. ${ }^{3}$ Based on this aging mechanism, antioxidant agents might add a potential therapeutic value in treating aging-associated diseases.

It is important to select a safe and effective antioxidant for alleviating oxidative stress to prevent and/or delay aging and aging-related diseases. Molecular hydrogen 
$\left(\mathrm{H}_{2}\right)$ is a colorless and odorless gas with a small molecular weight. In 2007, Ohsawa et al demonstrated that $\mathrm{H}_{2}$ could react with strong oxidants such as hydroxyl radical in cells. ${ }^{4}$ Since then, several researchers have confirmed the therapeutic and preventive effects of $\mathrm{H}_{2}$ in various clinical trials and animal disease models. ${ }^{5-7} \mathrm{H}_{2}$ therapy has been applied in aging-related diseases, such as type 2 diabetes, atherosclerosis, Parkinson's disease, and Alzheimer's disease. ${ }^{6-8} \mathrm{H}_{2}$ can be administered via different routes such as $\mathrm{H}_{2}$ gas inhalation, ${ }^{5} \mathrm{H}_{2}$-rich water (HRW) drinking, ${ }^{9} \mathrm{H}_{2}$-rich saline (HRS) injection, ${ }^{10}$ and direct incorporation of $\mathrm{H}_{2}$ through diffusion (such as taking $\mathrm{H}_{2}$ water bath ${ }^{11}$ or dropping $\mathrm{H}_{2}$-saline into the eyes). ${ }^{12}$ Previous studies have mainly focused on the effectiveness of $\mathrm{H}_{2}$ administered via a specific route. It is of great importance to explore the best administration method for different diseases. In addition, the functions of $\mathrm{H}_{2}$ in aging have not been deeply explored.

The D-galactose (D-Gal)-induced accelerated aging model is commonly used to study the pathological characteristics of senescence and to screen antiaging candidates ${ }^{13} \mathrm{D}$-Gal overload accelerates the accumulation of ROS, resulting in oxidative stress. This further increases the levels of free radicals, decreases antioxidant activity, and aggravates oxidative stress in the liver, brain, heart, kidney, and lung. ${ }^{13,14}$ The D-Gal exposure can exacerbate oxidative damage, including increased level of lipid peroxidation (LPO), and decreased activity of superoxide dismutase (SOD). ${ }^{13}$ In the meantime, the D-gal-induced oxidative damage drives aging-related disorders, accompanied by the formation of advanced glycation end products (AGEs) due to the glycation, oxidation, and/or carbonylation of proteins or lipids. ${ }^{15}$ As the chronic proinflammatory status is a pervasive feature of aging, it is recognized that the levels of the pro-inflammatory cytokines are elevated, such as tumor necrosis factor (TNF)$\alpha{ }^{16}$ Aging is accompanied by a decline in physiological function of many tissues and organs. This may lead to the increased free fatty acids (FFA) release from adipose tissue, ${ }^{17}$ and the elevated markers of organ damage, including the liver damage marker of alanine aminotransferase (ALT). ${ }^{18}$

The present study aimed to compare the different routes of $\mathrm{H}_{2}$ administration and investigate the protective effects of $\mathrm{H}_{2}$ on oxidative stress in D-Gal-induced aging mice. This may provide an experimental basis for its potential clinical applications against aging.

\section{Materials and Methods}

\section{Animals and Study Design}

Six-week-old C57BL/6J male mice (Vital River Laboratory Animal Technology Co., Ltd., Beijing, China) were housed in a controlled environment ( $12 \mathrm{~h}$ per day/night cycle, temperature $22^{\circ} \mathrm{C} \pm 1{ }^{\circ} \mathrm{C}$, humidity $50-60 \%$ ) with free access to a standard chow diet and water. All animal care and experimental procedures were approved by the laboratory animal ethics committee of Shandong First Medical University (Approve No. W202107080305). All the procedures were performed in accordance with the guidelines of the laboratory animal ethics committee of Shandong First Medical University. After 1 week of acclimatization, mice were randomly assigned to six groups $(\mathrm{n}=10$ /group): Control, Model, $4 \% \mathrm{H}_{2}$, HRW, HRS, and Ator groups. Except for the mice in the Control group, all mice were injected subcutaneously with $200 \mathrm{mg} / \mathrm{kg}$ bw D-Gal (SigmaAldrich, St. Louis, MO, USA) once a day. Mice in the $4 \% \mathrm{H}_{2}, \mathrm{HRW}$, and $\mathrm{HRS}$ groups were given $4 \% \mathrm{H}_{2^{-}}$ inhalation, HRW drinking, and HRS injection, respectively. As the antioxidative, anti-inflammatory, and anti-aging properties of atorvastatin have been proved, mice in the Ator group were intragastrically administered with atorvastatin $(0.1 \mathrm{mg} / \mathrm{kg} \mathrm{bw} / \mathrm{d})$ and served as the positive control. ${ }^{18,19}$ The Control group was given the same volume of physiological saline.

Mice were sacrificed after 10 weeks of administration. After starved for $12 \mathrm{~h}$, blood samples were collected in EDTA-coated tubes from the orbital sinus under isoflurane anesthesia. Then the mice were sacrificed by cervical dislocation, and tissue samples were collected. Blood samples were centrifuged at $3000 \mathrm{rpm}$ for $15 \mathrm{~min}$ at $4^{\circ} \mathrm{C}$, and the plasma was stored at $-80^{\circ} \mathrm{C}$. The liver, thymus, and spleen of each mouse were carefully dissected out, washed with cold phosphate-buffered saline (PBS, pH 7.4), and weighed. The ratio of organ weight to the final body weight was calculated and set as the organ index $(\mathrm{mg} / \mathrm{g})$. A portion of the brain, liver, and heart tissues were rapidly excised, dipped in liquid nitrogen, and stored at $-80^{\circ} \mathrm{C}$ until further biochemical analysis. A portion of the liver tissues was fixed in $4 \%$ paraformaldehyde for $24 \mathrm{~h}$ for histopathological examination.

\section{$\mathrm{H}_{2}$ Administration}

$4 \% \mathrm{H}_{2}$ Inhalation

Mice in $4 \% \mathrm{H}_{2}$ group were given $4 \%(\mathrm{v} / \mathrm{v}) \mathrm{H}_{2}$ gas via our self-made device for $2 \mathrm{~h}$ once daily as previously 
described. ${ }^{20}$ Briefly, $\mathrm{H}_{2}$ from an $\mathrm{H}_{2}$ generator and air from an air generator were controlled using flow meters and mixed in a plastic box. The concentration of $\mathrm{H}_{2}$ in the mixed gas was confirmed using a $\mathrm{H}_{2}$ detector (XP-3140, New Cosmos Electric Co., Ltd., Japan). Mice in Control, Model, HRW, HRS, and Ator groups were provided with air from an air generator in a similar device simultaneously. $^{20}$

\section{HRW Drinking}

Mice were provided with HRW ad libitum. HRW was prepared in an HRW-producing apparatus (ZQLHF-150G, Zhongqinglianhe Tech Co., Ltd, Liaocheng, China), in which $\mathrm{H}_{2}$ was dissolved in pure water under a pressure of $0.6 \mathrm{MPa}$. $\mathrm{HRW}$ was stored in a drinking bottle with stainless steel balls in the outlet. The concentration of $\mathrm{H}_{2}$ in water was monitored using a Clark-type hydrogen microsensor (Unisense, Aarhus N, Denmark). HRW was changed every $8 \mathrm{~h}$ to ensure that the concentration of $\mathrm{H}_{2}$ is above $600 \mu \mathrm{mol} / \mathrm{L}$.

\section{HRS Injection}

Hydrogen-rich saline was prepared as described in our previous report. ${ }^{10}$ Briefly, $\mathrm{H}_{2}$ from an $\mathrm{H}_{2}$ generator was dissolved in saline under high pressure $(0.4 \mathrm{MPa})$ with the air removed. HRS was stored at $4{ }^{\circ} \mathrm{C}$ and freshly prepared every day to ensure a constant hydrogen concentration. The concentration of hydrogen was monitored using a Clark-type hydrogen microsensor (Unisense, Aarhus N, Denmark) and was found to be above $700 \mu \mathrm{mol} / \mathrm{L}$. HRS $(0.1 \mathrm{~mL} / 10 \mathrm{~g} \mathrm{bw} / \mathrm{day})$ was intraperitoneally injected once daily in mice.

\section{$\mathrm{H}_{2}$ Concentration Monitoring in Mice Liver}

Six-week-old C57BL/6J male mice were anesthetized for the entire hydrogen monitoring procedure by administering an intraperitoneal injection of $20 \%$ urethane $(7 \mathrm{~mL} /$ $\mathrm{kg}$ ). A Clark-type hydrogen microsensor (Unisense, Aarhus N, Denmark) with a sensing anode (tip diameter 40-60 $\mu \mathrm{m}$ ) was used to measure real-time $\mathrm{H}_{2}$ concentration in the liver. A standard curve was established by diluting $\mathrm{H}_{2}$-saturated $\mathrm{PBS}$ at $38^{\circ} \mathrm{C}$. The liver tissue was exposed and the tip of the microsensor was inserted at a depth of $1 \mathrm{~mm} .4 \% \mathrm{H}_{2}$ was prepared using a self-made gas mixing device as described above and the mixed gas was administered through a gas supply hood.

\section{Biochemical Analysis}

Plasma total antioxidant capacity (TAOC), ALT, and FFA levels were measured using commercial kits purchased from Nanjing Jiancheng Bioengineering Institute (Nanjing, China). AGEs and TNF-awere determined using ELISA kits (Enzyme-linked Biotechnology, Shanghai, China) according to the manufacturer's instructions.

Tissue samples of the liver, brain, and heart were homogenized $(10 \% \mathrm{w} / \mathrm{v})$ in icy PBS $(\mathrm{pH} 7.4)$ and then centrifuged at $3000 \mathrm{~g}$ for $10 \mathrm{~min}$ at $4^{\circ} \mathrm{C}$. The supernatants were collected and used for biochemical evaluation. SOD activity was determined using commercial kits according to the protocols (Nanjing Jiancheng Bioengineering Institute, Nanjing, China). LPO was determined using a reaction with N-methyl-2-phenylindole to yield a stable chromophore with maximal absorbance at $586 \mathrm{~nm}$ (Nanjing Jiancheng Bioengineering Institute, Nanjing, China). The level of malondialdehyde (MDA) was determined via spectrophotometric measurements of thiobarbituric acid-reactive substances (Beyotime Biotechnology, Shanghai, China). Protein concentration was determined using the bicinchoninic acid method with bovine serum albumin as the standard (Beyotime Biotechnology, Shanghai, China).

\section{Histological Examination}

Liver tissues in 4\% paraformaldehyde were processed with graded volumes of alcohol, embedded in paraffin, and sectioned into 5 - $\mu \mathrm{m}$-thick slices. Samples were stained with Hematoxylin and Eosin (H\&E) and viewed under an optical microscope (Olympus BX53F, Tokyo, Japan).

\section{Statistical Analyses}

Data were analyzed for normal distribution by the Kolmogorov-Smirnov test. Continuous parameters were normally distributed and presented as mean \pm standard deviation (SD). Differences among the groups were assessed using one-way analysis of variance followed by least significant difference post-hoc test. Differences were considered significant when $P<0.05$. All statistical analyses were performed using IBM SPSS Statistics for Windows, Version 20.0 (IBM SPSS Inc., Chicago, IL, USA).

\section{Results}

\section{$\mathrm{H}_{2}$ Intake Dose Following Different Routes}

Different routes of $\mathrm{H}_{2}$ administration result in different $\mathrm{H}_{2}$ intake doses and pharmacokinetics. After inhalation of $4 \%$ $\mathrm{H}_{2}$, the concentration of $\mathrm{H}_{2}$ raised rapidly and achieved 
A

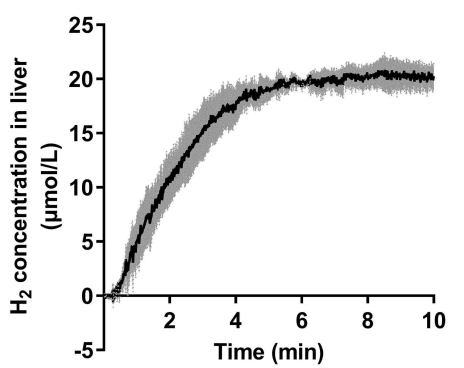

B

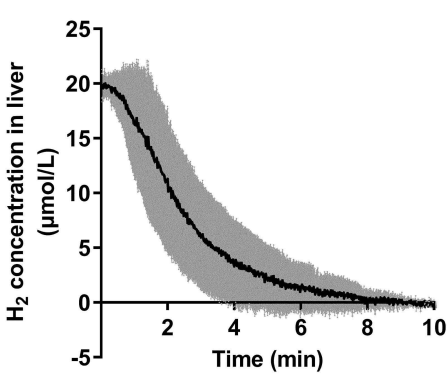

C

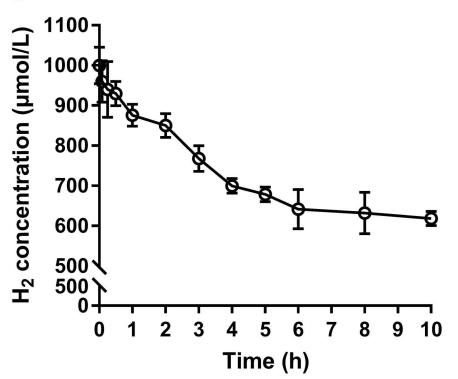

Figure $\mathrm{I} \mathrm{H}_{2}$ concentration measurement. (A) $\mathrm{H}_{2}$ concentration in the liver of mice during inhalation of $4 \% \mathrm{H}_{2}(\mathrm{n}=3)$, (B) $\mathrm{H}_{2}$ concentration in the liver of mice after inhalation of $4 \% \mathrm{H}_{2}(n=3)$, and $(\mathbf{C}) \mathrm{H}_{2}$ concentration of $\mathrm{HRW}$ in drinking bottled water $(n=3)$. Data are presented as mean $\pm \mathrm{SD}$.

equilibrium at $20 \mu \mathrm{mol} / \mathrm{L}$ in approximately 5 minutes in liver (Figure 1A). As $\mathrm{H}_{2}$ is a small gas molecule with strong penetrability, it is not preserved in the body for a long time. After withdrawing the administration of $\mathrm{H}_{2}$, its concentration in the liver exhibited a sharp drop and then the curve decreased gradually until it reached the baseline in approximately 8 minutes (Figure $1 \mathrm{~B}$ ). Also, we monitored $\mathrm{H}_{2}$ concentration of HRW in drinking bottles. Drinking HRW led to an intermittent access to $\mathrm{H}_{2}$. Supersaturated $\mathrm{H}_{2}$ in HRW (1000 $\mu \mathrm{mol} / \mathrm{L})$ was kept at a high concentration for a long time and stayed above $600 \mu \mathrm{mol} / \mathrm{L}$ even after 8 hours (Figure $1 \mathrm{C}$ ). In order to maintain a high $\mathrm{H}_{2}$ concentration, HRW was freshly prepared and replaced every 8 hours throughout the experiment. The water intake of mice was about $1.5 \mathrm{~mL} / 10 \mathrm{~g} \mathrm{bw} / \mathrm{day},{ }^{21,22}$ and thus the $\mathrm{H}_{2}$ intake with HRW was $0.6-1.5 \mu \mathrm{mol} / 10 \mathrm{~g}$ bw/day. For HRS injection, mice received $700 \mu \mathrm{mol} / \mathrm{L} \mathrm{HRS}$ for $0.1 \mathrm{~mL} / 10 \mathrm{~g} \mathrm{bw} /$ day, equal to a $\mathrm{H}_{2}$ dose of $0.07 \mu \mathrm{mol} / 10 \mathrm{~g}$ bw/day.

\section{Effect of $\mathrm{H}_{2}$ on Body Weight and Organ Index}

As shown in Table 1, after an 8-week exposure to D-gal, the Model group showed a significant decrease in body weight as compared with the Control group $(P<0.05)$. The liver and thymus indexes of the Model group decreased significantly as compared with the Control group $(P<0.001$ and $P<0.01$, respectively). The administration of $\mathrm{H}_{2}$ or atorvastatin did not improve the body weight significantly compared with the Model group $(P>0.05)$. Different $\mathrm{H}_{2}$ administration routes led to different effects on the organ indexes. The liver index with HRS injection and thymus index with $4 \% \mathrm{H}_{2}$ inhalation increased notably $(P<0.05)$, whereas HRW drinking significantly decreased the spleen index compared with the Model group $(P<0.05)$.

\section{$\mathrm{H}_{2}$ Improves Aging-Related Biomarkers in} Plasma

Aging is accompanied by the accumulation of ROS that causes oxidative damage. ${ }^{2}$ As shown in Figure 2A, plasma TAOC was significantly decreased in D-Gal-induced aging mice, indicating a decline in their antioxidant status. The administration of $\mathrm{H}_{2}$ via $4 \% \mathrm{H}_{2}$ inhalation, $\mathrm{HRW}$ drinking, and HRS injection significantly increased the levels of TAOC by $26.4 \%, 30.6 \%$, and $34.8 \%$, respectively. Compared with the Control, treatment with D-Gal was able to induce an increase of plasma AGEs, of which

Table I Effects of $\mathrm{H}_{2}$ on Body Weight and Organ Index in Mice

\begin{tabular}{|l|l|l|l|l|}
\hline \multirow{2}{*}{ Group } & Body Weight $(\mathbf{g})$ & \multicolumn{3}{|c|}{ Organ Index (mg/g) } \\
\cline { 3 - 5 } & & Liver Index & Thymus Index & Spleen Index \\
\hline Control & $26.7 \pm 0.97$ & $44.31 \pm 2.12$ & $2.07 \pm 0.34$ & $2.55 \pm 0.41$ \\
Model & $25.49 \pm 1.33^{*}$ & $41.71 \pm 1.66^{* * *}$ & $1.76 \pm 0.24^{* *}$ & $2.73 \pm 0.32$ \\
$4 \% \mathrm{H}_{2}$ & $26.09 \pm 1.48$ & $40.82 \pm 1.72$ & $1.95 \pm 0.22^{\#}$ & $2.64 \pm 0.32$ \\
HRW & $24.78 \pm 0.69$ & $43.22 \pm 1.73$ & $1.93 \pm 0.22$ & $2.48 \pm 0.19^{\#}$ \\
HRS & $25.98 \pm 2.17$ & $43.72 \pm 3.54^{\#}$ & $1.85 \pm 0.38$ & $2.77 \pm 0.56$ \\
Ator & $25.91 \pm 1.00$ & $42.97 \pm 4.54$ & $2.11 \pm 0.32^{\# \#}$ & $2.84 \pm 0.39$ \\
\hline
\end{tabular}

Notes: Values represent the mean $\pm S D, n=10 . * P<0.05, * * P<0.01$, and $* * * p<0.001$ compared with the Control group. ${ }^{\#} p<0.05$ and ${ }^{\# \#} p<0.01$ compared with the Model group. 

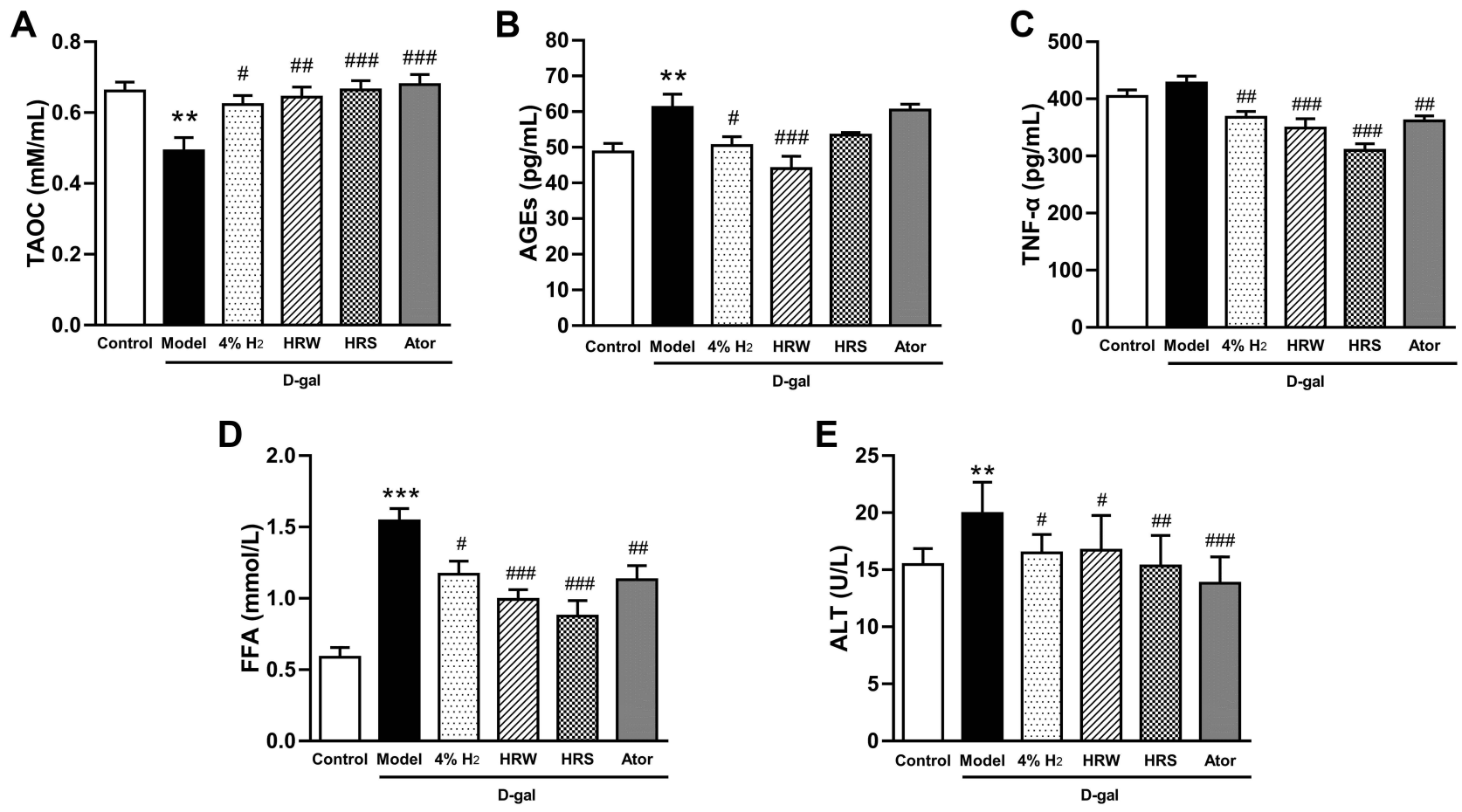

Figure 2 Effects of $\mathrm{H}_{2}$ on aging-related biomarkers in plasma of D-gal-induced aging mice. (A) TAOC, (B) AGEs, (C) TNF- $\alpha$, (D) FFA, and (E) ALT levels. Data are expressed as mean \pm SD $(n=10)$. ${ }^{* * P}<0.01$, ${ }^{* * * P} P 0.001$ compared with the Control group; ${ }^{\#} P<0.05$, ${ }^{\# \#} P<0.01$, and ${ }^{\# \#} P<0.001$ compared with the Model group.

a biomarker implicated in aging $(P<0.01$, Figure 2B).

The levels of AGEs after $4 \% \mathrm{H}_{2}$ and HRW administration were $17.4 \%$ and $28.0 \%$ lower than those of the Model group $(P<0.05$ and $P<0.01)$, respectively. The level of AGEs also showed a decreasing trend after treatment with HRS injection $(P=0.11)$. Development of chronic inflammation is a hallmark of oxidative damage accumulation as the inflammatory processes contribute to cellular dysfunctions and tissue damage. As shown in Figure $2 \mathrm{C}$, different $\mathrm{H}_{2}$ treatment methods significantly reduced the level of TNF- $\alpha$, an inflammation-associated indicator. A high FFA level is associated with inflammatory states and agingrelated diseases, such as vascular endothelial disorders, insulin resistance, and diabetes. ${ }^{23} \mathrm{D}$-Gal treated mice showed a significant increase in plasma FFA, and administration of $\mathrm{H}_{2}\left(4 \% \mathrm{H}_{2}\right.$ inhalation, HRW drinking, and HRS injection) significantly decreased the levels of FFA $(P<0.05, P<0.001$, and $P<0.001$, respectively, Figure 2D). As a biochemical parameter of liver function, the level plasma ALT increased in the Model group in comparison with that in the Control group $(P<0.01)$, and administration of $\mathrm{H}_{2}$ attenuated ALT elevation induced by D-Gal $\left(P<0.05, P<0.05\right.$, and $P<0.01$ for $4 \% \mathrm{H}_{2}$ inhalation, HRW drinking, and HRS injection, respectively) (Figure 2E).

\section{$\mathrm{H}_{2}$ Attenuates D-Gal-Induced Oxidative}

\section{Stress in the Liver, Brain, and Heart}

We next examined the antioxidative effects of $\mathrm{H}_{2}$ on different organs. The free radical chain reaction of lipid results in the production of LPO. MDA is the most stable breakdown product of LPO and is also regarded as a possible marker of aging. ${ }^{24}$ LPO and MDA levels in liver (Figure 3A and $\mathrm{B}$ ), brain (Figure 3D and $\mathrm{E}$ ), and heart (Figure 3G and $\mathrm{H}$ ) of mice in the Model group were significantly higher than those in different organs of mice in the Control group. All $\mathrm{H}_{2}$ administration methods significantly decreased the hepatic LPO and MDA levels. In addition, $4 \% \mathrm{H}_{2}$ inhalation showed statistically significant effects on LPO and MDA levels in the brain and heart, whereas HRW drinking significantly reduced the LPO level in the heart. However, HRS injection did not demonstrate any significant effect on the levels of LPO and MDA in the brain and heart. The activity of the antioxidant enzyme SOD has been reported to decrease in the aging process. ${ }^{25}$ As shown in Figure $3 \mathrm{C}$ and F, mice treated with D-Gal showed a significant decrease in SOD activities in the liver and brain as compared with mice in the Control group $(P<0.001$ and $P<0.05$, respectively). Treatment with $\mathrm{H}_{2}$ or atorvastatin was able to significantly increase the activities of SOD in the same organs. In this 
A

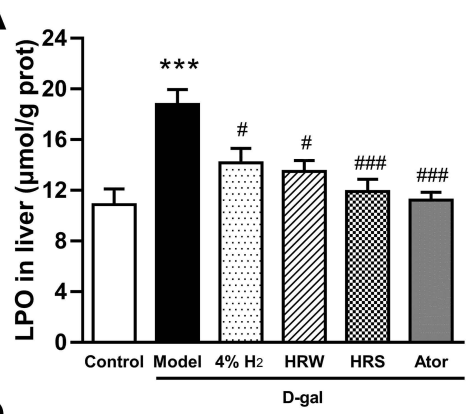

D

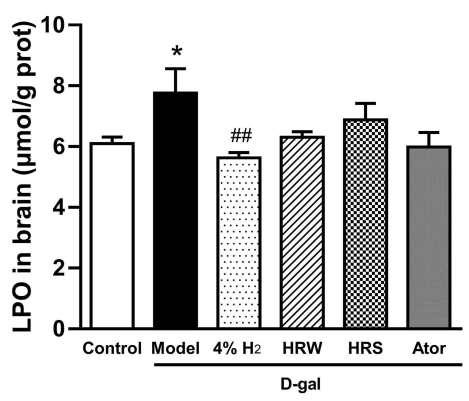

G

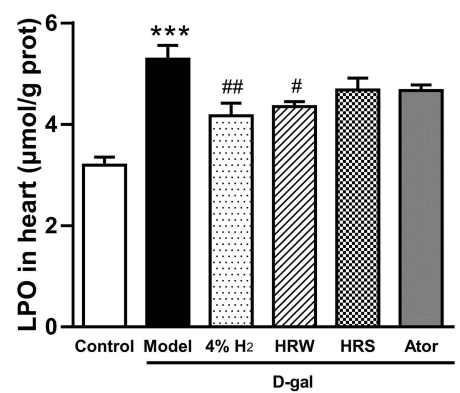

B

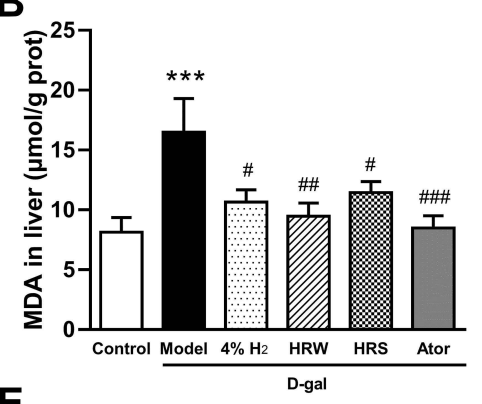

E

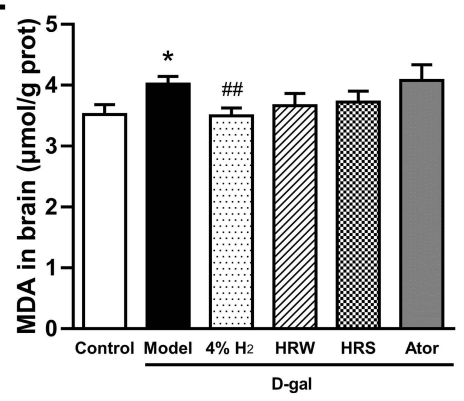

H

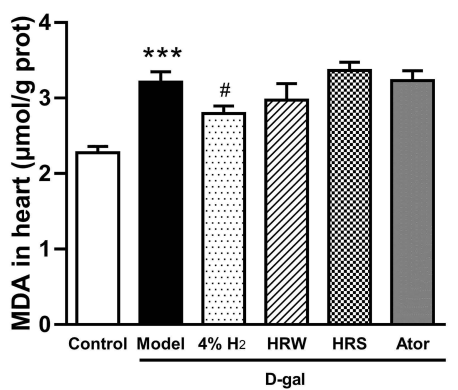

C

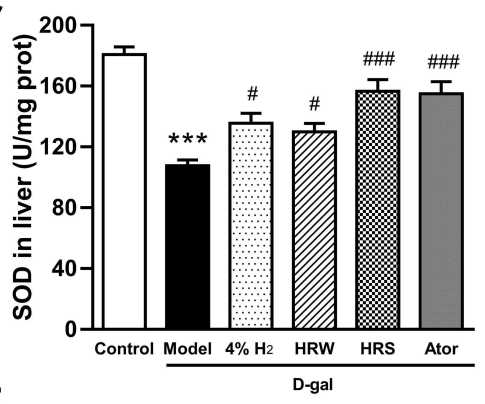

E

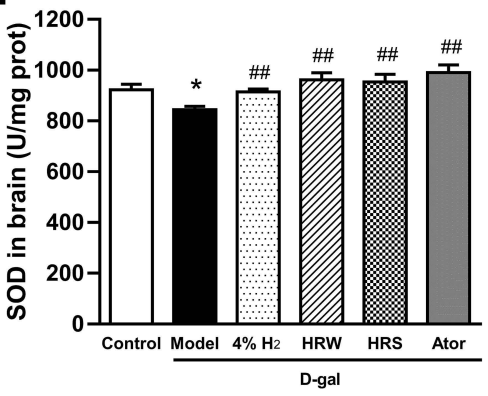

I

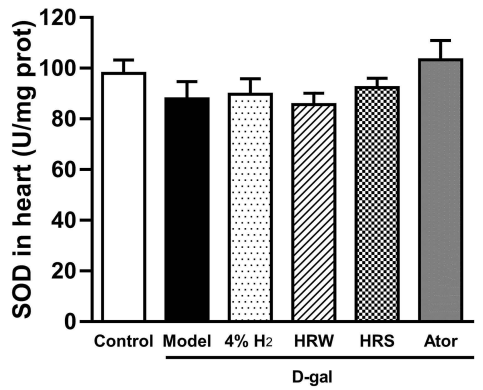

Figure $3 \mathrm{H}_{2}$ alleviates oxidative stress in D-Gal-induced aging mice. LPO levels, MDA levels, and SOD activities in the liver (A-C), brain (D-F), and heart (G-I). Data are expressed as mean \pm SD $(n=10)$. ${ }^{*} P<0.05$, $* * * P<0.001$ compared with the Control group; ${ }^{\#} P<0.05$, ${ }^{\#} P<0.01$, and ${ }^{\# \#} P<0.001$ compared with the Model group.

experiment, D-Gal or $\mathrm{H}_{2}$ treatment did not show any significant effects on the SOD activity in the heart $(P>$ 0.05 , Figure 3I).

\section{Histological Analyses of Liver}

D-Gal is mainly metabolized in the liver. Oxidative stress damage and inflammatory response induced by D-Gal can result in cell death and consequent damage to hepatic functions. ${ }^{13}$ The histological features of liver sections were illustrated by $H \& E$ staining. Hepatic tissues from the Control group showed normal architecture with a regular arrangement of hepatic cells radially around the central vein (Figure 4A). In contrast, D-gal treatment resulted in a disordered arrangement of hepatocytes with infiltration of inflammatory cells and hepatocytes necrosis (Figure 4B). The hepatic pathological alterations were notably ameliorated by $\mathrm{H}_{2}$ or atorvastatin treatment.
Liver cells of the mice in the Control group showed a more orderly arrangement compared with those of the mice in the Model group, while hepatocyte inflammation and necrosis were reduced across all treatments (Figure 4C-F).

\section{Discussion}

In this study, we demonstrated that the administration of $\mathrm{H}_{2}$ can alleviate oxidative stress in D-Gal-induced aging mice, and the effects of different $\mathrm{H}_{2}$-administration routes may vary. We proposed that $\mathrm{H}_{2}$ may serve as an antiaging intervention partly through its antioxidative properties.

As an effective medical gas with great biosafety, $\mathrm{H}_{2}$ has been attracting an increasing interest. Several studies have suggested that $\mathrm{H}_{2}$ may suppress the progress of aging. Klichko et al reported that supplementation with $\mathrm{H}_{2}$-rich food prolonged the life span in Drosophila. ${ }^{26}$ 

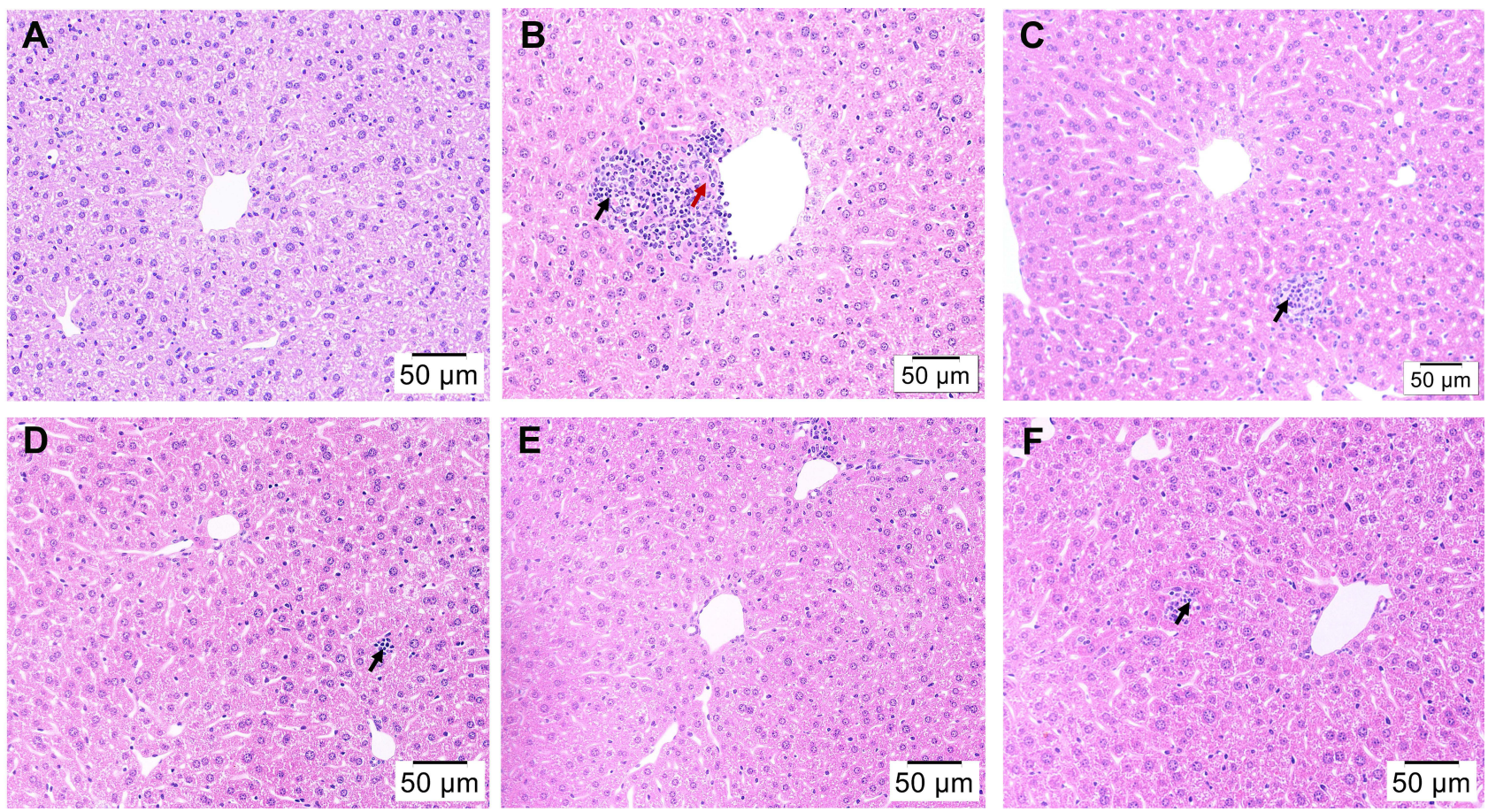

Figure 4 Effect of $\mathrm{H}_{2}$ on liver histopathological alterations in mice injured by D-Gal (H\&E staining, magnification 200x). (A) Control group, (B) Model group, (C) $4 \% \mathrm{H}_{2}$ group, (D) HRW group, (E) HRS group, and (F) Ator group. Black arrows indicate inflammatory cell infiltration and red arrow indicates necrosis.

A hallmark of aging is the chronic accumulation of cellular senescence in aged organisms, which contributes to tissue dysfunction and aging-related phenotypes. ${ }^{27}$ A previous report showed that drinking HRW could decrease the number of endothelial cells in an atheroma expressing the senescence factors p16INK4a and p21 in high-fat diet-fed low-density lipoprotein receptor-deficient $\left(\mathrm{Ldll}^{-1}\right)$ mice. ${ }^{9}$ AGEs are a heterogeneous group of molecules generated through nonenzymatic glycation and oxidation of proteins, lipids, and nucleic acids and can result in various aging-related disorders. A recent study showed that orally administered HRW had the potential to decrease AGEs and the expression of their receptors in streptozotocin-induced diabetic rats. ${ }^{28}$ The antioxidant property of $\mathrm{H}_{2}$ has been demonstrated against hydroxyl radicals $(\cdot \mathrm{OH})$ and peroxynitrite $\left(\mathrm{ONOO}^{-}\right){ }^{4}$ In this study, we studied the effects of $\mathrm{H}_{2}$ on free radical damage in D-Galinduced accelerated aging model.

D-Gal-induced oxidation-related damage includes the decreased activity of antioxidant enzymes, increased generation of protein carbonyls and lipid peroxides, inflammation, apoptosis, and DNA fragmentation. ${ }^{13}$ Besides the well-recognized lipid lowering effect, the antioxidative, anti-inflammatory, and anti-aging properties of atorvastatin were also established. ${ }^{18,19}$ Thus, mice intragastrically administered with atorvastatin served as the positive control. The antioxidative effects of $\mathrm{H}_{2}$ have been reported in several studies. ${ }^{6}$ We previously observed that the intraperitoneal injection of HRS decreased serum oxidized lowdensity lipoprotein level and reduced ROS accumulation in the aorta in an atherosclerosis model of $\mathrm{Ldlr}^{-/-}$mice. ${ }^{29}$ Furthermore, we demonstrated that inhalation of $4 \% \mathrm{H}_{2}$ decreased the concentration of oxidized phospholipids in rats fed with a high-fat diet. ${ }^{20}$ In the present study, free radical-mediated formation of LPO and MDA decreased, whereas the activity of the antioxidant enzyme SOD increased in targeted organs after $\mathrm{H}_{2}$ treatment. These results strongly suggested that $\mathrm{H}_{2}$ presents preventive effects against oxidative stress in D-Gal-induced aging mice. Plasma aging indicators, such as AGEs, antioxidant ability of TAOC, inflammatory cytokine TNF- $\alpha$, and liver injury marker ALT, all improved upon $\mathrm{H}_{2}$ treatment. These results suggested the preventive and therapeutic potential of $\mathrm{H}_{2}$ as an effective antiaging intervention.

There are several possible mechanisms of action. The most influential hypothesis is that $\mathrm{H}_{2}$ acts as a therapeutic antioxidant by reacting with cytotoxic ROS and thus protecting against oxidative damage. ${ }^{4}$ Our results showed that a very small amount of $\mathrm{H}_{2}$ administrated by HRW drinking or HRS injection are comparable to $4 \% \mathrm{H}_{2}$ inhalation in 
many indicators. Whether exogenous $\mathrm{H}_{2}$ exerts its effects as a gaseous signal molecule is still uncertain. Recently, a study provided the evidence for the direct interaction between $\mathrm{H}_{2}$ with proteins. Thus, $\mathrm{H}_{2}$ may directly influence the enzymatic activity and protein structure. Whether $\mathrm{H}_{2}$ can affect the antioxidant enzymes needs to be explored in depth.

Changes of organ indexes in mice have important reference value for analyzing mouse aging and the effects of antiaging candidates. The effects of various $\mathrm{H}_{2}$ administration routes on diverse organ indexes were different. In order to explain the underlined effects of $\mathrm{H}_{2}$, further experimental research is needed.

Dosages and routes of drug administration play an influential role in its efficiency. Three routes of $\mathrm{H}_{2}$ administration were compared in the present study. After $\mathrm{H}_{2}$ inhalation, $\mathrm{H}_{2}$ enters the arterial blood through the alveoli and is then taken into tissues by a pressure gradient. ${ }^{30} \mathrm{H}_{2}$ is maintained at a relative saturated level in circulation and tissues based on the $\mathrm{H}_{2}$ concentration inhaled. It is worth noting that the $\mathrm{H}_{2}$ concentrations of different tissues showed different saturation rates. A previous study showed that with $2 \% \mathrm{H}_{2}$ gas inhalation, the arterial and myocardium $\mathrm{H}_{2}$ levels reached a maximum level after 5 min; by contrast, the $\mathrm{H}_{2}$ saturation rate was much slower in the center of the thigh muscle with attaining a maximum level after 30 min. $^{31}$ The current study revealed that the equilibrium was reached quickly in liver within $5 \mathrm{~min}$. The equilibrium time of $\mathrm{H}_{2}$ in other tissues and organs needs to be studied further in detail. Oral $\mathrm{H}_{2}$ from $\mathrm{HRW}$ is in direct contact with the gastrointestinal tract. This is a convenient and the most widely applied method to study the uptake of $\mathrm{H}_{2}$. For intraperitoneal injection of HRS, the dissolved $\mathrm{H}_{2}$ spreads into the abdominal organs. Here, the $\mathrm{H}_{2}$ uptake can be accurately controlled. We evaluated the $\mathrm{H}_{2}$ concentration in the liver during and after $\mathrm{H}_{2}$ inhalation. However, as HRW drinking or HRS injection provided a relatively small amount of $\mathrm{H}_{2}$ and it is extremely easy to escape, we could not detect the accurate $\mathrm{H}_{2}$ concentration in vivo.

The dose-response relation in $\mathrm{H}_{2}$ has not been well studied. Inhalation of hydrogen-oxygen mixture $\left(66 \% \mathrm{H}_{2}\right.$ $133 \% \mathrm{O}_{2}$ ) produced by electrolyzing water proved to benefit cancer patients. ${ }^{32}$ Several studies have suggested that inhalation of low concentrations of $\mathrm{H}_{2}(1-4 \%)$ could protect cells and tissues against oxidative stress. ${ }^{4,20}$ Similarly, small amounts of oral $\mathrm{H}_{2}$-water are sufficient for gastric induction of ghrelin and exert a neuroprotective effect. ${ }^{33}$
The current study revealed that although the various $\mathrm{H}_{2}$ administration methods led to different dosages and action times, they all exerted an antioxidant effect. Among them, $4 \% \mathrm{H}_{2}$ inhalation decreased the accumulation of lipid peroxides in the brain. Compared with $\mathrm{H}_{2}$ inhalation, oral $\mathrm{H}_{2}$-water and intraperitoneal injection of $\mathrm{H}_{2}$-saline resulted in limited $\mathrm{H}_{2}$ intake. It is worth noting that although the amount of $\mathrm{H}_{2}$ taken up by HRW was reduced, compared to that of $\mathrm{H}_{2}$ gas inhalation, it resulted in the same efficacy in most indicators. Compared with a certain period of $\mathrm{H}_{2}$ inhalation or given amounts of $\mathrm{H}_{2}$ by HRS injection, drinking HRW leads an intermittent source of $\mathrm{H}_{2}$. A previous report revealed that intermittent hydrogen gas exposure could prevent 6-hydroxydopamine-induced Parkinson's disease in rats. ${ }^{34}$ Additional research is required to further confirm the biological effects of $\mathrm{H}_{2}$ via different administration routes.

This study compared three different routes; however, the doses chosen are also different. In addition, the doseresponse effect for a specific route of administration of $\mathrm{H}_{2}$ has not been the focus of our research in this study. The dose-response effect has been observed in $\mathrm{H}_{2}$ gas inhalation for the treatment of ischemia-reperfusion injury. ${ }^{31,35}$ Lower concentrations of $\mathrm{H}_{2}(0.5-2 \%)$ provided better effects than $4 \% \mathrm{H}_{2}$ inhalation. ${ }^{31}$ Therefore, further investigation is necessary to identify an optimal $\mathrm{H}_{2}$ dose.

There still exist some limitations in the study. The aging-related parameters measured were insufficient and a more comprehensive study is needed to verify the effects of $\mathrm{H}_{2}$. We measured the LPO, MDA, and SOD levels in liver, heart, and brain, as these tissues are most agingrelated. Other tissues such as kidney, muscle, skin can be further studied. Moreover, we measured the aging-related biomarkers of TAOC, AGEs, TNF- $\alpha$, and FFA in plasma. The more specific aging-related markers in tissues are worthy of in-depth study. In addition, we only selected male mice as the experimental subjects; however, the female subjects should also be included as genderspecific differences.

This study provides support to an effective anti-aging intervention of $\mathrm{H}_{2}$ administration by mitigating oxidative stress in aging. It is known that $\mathrm{H}_{2}$ administrations via different routes such as $\mathrm{H}_{2}$ gas inhalation, $\mathrm{H}_{2}$-rich water drinking, intravenous $\mathrm{H}_{2}$-saline infusion, and $\mathrm{H}_{2}$-rich water bath have been applied in different clinical trials. Owning to the great biosafety of $\mathrm{H}_{2}$, it will be of great interest to translate our study to humans. 


\section{Conclusion}

In conclusion, $\mathrm{H}_{2}$ can prevent oxidative stress in D-Galinduced accelerated aging model of mice when administered by different routes. Among the different routes of $\mathrm{H}_{2}$ administration, $4 \% \mathrm{H}_{2}$ inhalation was the most effective in reducing the markers of oxidative stress in some of the tissues, however the other routes resulted in the same efficacy in most indicators. $\mathrm{H}_{2}$ treatment may be a promising antiaging intervention.

\section{Acknowledgments}

This work was supported by the National Natural Science Foundation of China [grant number 81770855], Taishan Scholars Program of Shandong Province [grant number ts201511057], Shandong Provincial Natural Science Foundation (ZR2020QH020), and Academic Promotion Programme of Shandong First Medical University [grant number 2019QL010, 2019PT009].

\section{Disclosure}

The authors report no conflicts of interest in this work.

\section{References}

1. López-Otín C, Galluzzi L, Freije JMP, et al. Metabolic control of longevity. Cell. 2016;166(4):802-821. doi:10.1016/j.cell.2016.07.031

2. Liochev SI. Reactive oxygen species and the free radical theory of aging. Free Radic Biol Med. 2013;60:1-4. doi:10.1016/j. freeradbiomed.2013.02.011

3. Schieber M, Chandel NS. ROS function in redox signaling and oxidative stress. Curr Biol. 2014;24(10):R453-R462. doi:10.1016/j. cub.2014.03.034

4. Ohsawa I, Ishikawa $M$, Takahashi $K$, et al. Hydrogen acts as a therapeutic antioxidant by selectively reducing cytotoxic oxygen radicals. Nat Med. 2007;13(6):688-694. doi:10.1038/nm1577

5. Liu B, Xue J, Zhang M, et al. Hydrogen inhalation alleviates nonalcoholic fatty liver disease in metabolic syndrome rats. Mol Med Rep. 2020;22(4):2860-2868. doi:10.3892/mmr.2020.11364

6. Tao G, Song G, Qin S. Molecular hydrogen: current knowledge on mechanism in alleviating free radical damage and diseases. Acta Biochim Biophys Sin. 2019;51(12):1189-1197. doi:10.1093/abbs/ gmz121

7. Chen W, Zhang HT, Qin SC. Neuroprotective effects of molecular hydrogen: a critical review. Neurosci Bull. 2021;37(3):389-404. doi:10.1007/s12264-020-00597-1

8. Qin S. Role of hydrogen in atherosclerotic disease: from bench to bedside. Curr Pharm Des. 2021;27(5):713-722. doi:10.2174/13816 12826666201124112152

9. Iketani $\mathrm{M}$, Sekimoto $\mathrm{K}$, Igarashi $\mathrm{T}$, et al. Administration of hydrogen-rich water prevents vascular aging of the aorta in LDL receptor-deficient mice. Sci Rep. 2018;8(1):16822. doi:10.1038/ s41598-018-35239-0

10. Song G, Tian H, Qin S, et al. Hydrogen decreases athero-susceptibility in apolipoprotein B-containing lipoproteins and aorta of apolipoprotein E knockout mice. Atherosclerosis. 2012;221 (1):55-65. doi:10.1016/j.atherosclerosis.2011.11.043
11. Zhu Q, Wu Y, Li Y, et al. Positive effects of hydrogen-water bathing in patients of psoriasis and parapsoriasis en plaques. Sci Rep. 2018;8 (1):8051. doi:10.1038/s41598-018-26388-3

12. Oharazawa H, Igarashi T, Yokota T, et al. Protection of the retina by rapid diffusion of hydrogen: administration of hydrogen-loaded eye drops in retinal ischemia-reperfusion injury. Invest Ophthalmol Vis Sci. 2010;51(1):487-492. doi:10.1167/iovs.09-4089

13. Azman KF, Zakaria R. D-galactose-induced accelerated aging model: an overview. Biogerontology. 2019;20(6):763-782. doi:10.1007/ s10522-019-09837-y

14. Wei H, Li L, Song Q, Ai H, Chu J, Li W. Behavioural study of the D-galactose induced aging model in C57BL/6J mice. Behav Brain Res. 2005;157(2):245-251. doi:10.1016/j.bbr.2004.07.003

15. Song X, Bao M, Li D, Li YM. Advanced glycation in D-galactose induced mouse aging model. Mech Ageing Dev. 1999;108(3):2 39-251. doi:10.1016/s0047-6374(99)00022-6

16. Franceschi $\mathrm{C}$, Campisi J. Chronic inflammation (inflammaging) and its potential contribution to age-associated diseases. J Gerontol A Biol Sci Med Sci. 2014;69(Suppl 1):S4-S9. doi:10.1093/gerona/glu057

17. Toth MJ, Tchernof A. Lipid metabolism in the elderly. Eur J Clin Nutr. 2000;54(Suppl 3):S121-S125. doi:10.1038/sj.ejen.1601033

18. Taghipour Z, Kaviani E, Kaeidi A, et al. Atorvastatin attenuates D-galactose-induced hepatorenal toxicity in mice: an experimental study with histopathological evaluations. Physiol Pharmacol. 2019; 23(1):36-43.

19. Kaviani E, Rahmani M, Kaeidi A, et al. Protective effect of atorvastatin on d-galactose-induced aging model in mice. Behav Brain Res. 2017;334:55-60. doi:10.1016/j.bbr.2017.07.029

20. Liu B, Zhao M, Xue J, Gu Q, Zhang X, Qin S. Hydrogen influences HDL-associated enzymes and reduces oxidized phospholipids levels in rats fed with a high-fat diet. Life Sci. 2021;267:118945. doi:10. 1016/j.lfs.2020.118945

21. Nicolaus ML, Bergdall VK, Davis IC, Hickman-Davis JM. Effect of ventilated caging on water intake and loss in 4 strains of laboratory mice. J Am Assoc Lab Anim Sci. 2016;55(5):525-533.

22. Middaugh LD, Kelley BM, Bandy AL, McGroarty KK. Ethanol consumption by C57BL/6 mice: influence of gender and procedural variables. Alcohol. 1999;17(3):175-183. doi:10.1016/s0741-8329(98) 00055-x

23. Miedema MD, Maziarz M, Biggs ML, et al. Plasma-free fatty acids, fatty acid-binding protein 4 , and mortality in older adults (from the Cardiovascular Health Study). Am J Cardiol. 2014;114(6):843-848. doi:10.1016/j.amjcard.2014.06.012

24. Gil P, Fariñas F, Casado A, López-Fernández E. Malondialdehyde: a possible marker of ageing. Gerontology. 2002;48(4):209-214. doi: $10.1159 / 000058352$

25. Inal ME, Kanbak G, Sunal E. Antioxidant enzyme activities and malondialdehyde levels related to aging. Clin Chim Acta. 2001;305 (1-2):75-80. doi:10.1016/s0009-8981(00)00422-8

26. Klichko VI, Safonov VL, Safonov MY, Radyuk SN. Supplementation with hydrogen-producing composition confers beneficial effects on physiology and life span in Drosophila. Heliyon. 2019;5(5):e01679. doi:10.1016/j.heliyon.2019.e01679

27. Cai Y, Zhou H, Zhu Y, et al. Elimination of senescent cells by $\beta$ galactosidase-targeted prodrug attenuates inflammation and restores physical function in aged mice. Cell Res. 2020;30(7):574-589. doi:10.1038/s41422-020-0314-9

28. Retnaningtyas E, Susatia B, Indrajani O, et al. Hydrogen-rich water supplementation declines advanced glycation-end products (AGE) and receptor for AGE (RAGE) in streptozotocin-induced diabetic rats. AIP Conf Proc. Vol. 2353. Issue (1). AIP Publishing LLC; 2021:030086.

29. Song G, Zong C, Zhang Z, et al. Molecular hydrogen stabilizes atherosclerotic plaque in low-density lipoprotein receptor-knockout mice. Free Radic Biol Med. 2015;87:58-68. doi:10.1016/j. freeradbiomed.2015.06.018 
30. Sun X, Ohta S, Nakao A. Hydrogen Molecular Biology and Medicine. Springer; 2015.

31. Hayashida K, Sano M, Ohsawa I, et al. Inhalation of hydrogen gas reduces infarct size in the rat model of myocardial ischemia-reperfusion injury. Biochem Biophys Res Commun. 2008;373(1):30-35. doi:10.1016/j.bbrc.2008.05.165

32. Chen JB, Kong XF, Lv YY, et al. "Real world survey" of hydrogencontrolled cancer: a follow-up report of 82 advanced cancer patients. Med Gas Res. 2019;9(3):115-121. doi:10.4103/2045-9912.266985

33. Matsumoto A, Yamafuji M, Tachibana T, et al. Oral "hydrogen water" induces neuroprotective ghrelin secretion in mice. Sci Rep. 2013;3(1):3273. doi:10.1038/srep03273
34. Ito M, Hirayama M, Yamai K, et al. Drinking hydrogen water and intermittent hydrogen gas exposure, but not lactulose or continuous hydrogen gas exposure, prevent 6-hydorxydopamine-induced Parkinson's disease in rats. Med Gas Res. 2012;2(1):1-7. doi:10.1186/2045-9912-2-15

35. Fukuda $\mathrm{K}$, Asoh $\mathrm{S}$, Ishikawa $\mathrm{M}$, et al. Inhalation of hydrogen gas suppresses hepatic injury caused by ischemia/reperfusion through reducing oxidative stress. Biochem Biophys Res Commun. 2007;361 (3):670-674. doi:10.1016/j.bbrc.2007.07.088

\section{Publish your work in this journal}

The Journal of Inflammation Research is an international, peerreviewed open-access journal that welcomes laboratory and clinical findings on the molecular basis, cell biology and pharmacology of inflammation including original research, reviews, symposium reports, hypothesis formation and commentaries on: acute/chronic inflammation; mediators of inflammation; cellular processes; molecular mechanisms; pharmacology and novel anti-inflammatory drugs; clinical conditions involving inflammation. The manuscript management system is completely online and includes a very quick and fair peerreview system. Visit http://www.dovepress.com/testimonials.php to read real quotes from published authors. 